\title{
Start up of biological sequencing batch reactor (SBR) and short-term biomass acclimation for polyhydroxyalkanoates production
}

\author{
Francesco Valentino, ${ }^{\text {a* }}$ Angelo Antonio Brusca, ${ }^{a}$ Mario Beccari, ${ }^{a}$ \\ Andrea Nuzzo, ${ }^{b, c}$ Giulio Zanaroli $^{b, c}$ and Mauro Majone ${ }^{a}$
}

\begin{abstract}
BACKGROUND: The adaptation/selection of mixed microbial cultures under feast/famine conditions is an essential step for polyhydroxyalkanoates (PHA) production. This study investigated the short-term adaptation of a mixed microbial culture (activated sludge) during the start up of a sequencing batch reactor (SBR).

RESULTS: Four different SBR runs were performed starting from different inocula and operated at the same organic load rate (8.5 gCOD L $\mathrm{L}^{-1} \mathrm{~d}^{-1}$ ) and hydraulic retention time (1 day). At 3-7 days from SBR start up, the selected biomass was able to store PHA at comparable rate and yield with those obtained after long-term acclimation. Independently from the time passed, a short feast phase was the key parameter to obtain PHA storage at high rate and yield in the following accumulation stage (244 $\mathrm{mgCOD} \mathrm{g}^{-1}$ CODnonPolym $\mathrm{h}^{-1}$ for specific storage rate and $48 \%$ COD COD ${ }^{-1}$ as PHA content in the biomass). The DGGE profiles showed that the good storage performance and the structure of the microbial community were not fully correlated.
\end{abstract}

CONCLUSIONS: The results suggest a new strategy for operating the PHA accumulation stage directly in the SBR, after very short biomass adaptation, instead of using two separate reactors for biomass enrichment and PHA accumulation, respectively. (c) 2012 Society of Chemical Industry

Keywords: polyhydroxyalkanoates (PHA); storage response; sequencing batch reactor (SBR); acclimation; nitrogen limitation; denaturing gradient gel electrophoresis (DGGE)

\section{INTRODUCTION}

In recent years, a lot of research has been dedicated to the production of biodegradable polymers (e.g. polyhydroxyalkanoates, PHA) from renewable resources: fermented sugar cane molasses, ${ }^{1,2}$ fermented palm oil mill effluents, $\mathrm{POME}^{3,4}$ fermented paper mill wastewater, ${ }^{5}$ tomato cannery waste, ${ }^{6}$ fermented brewery wastewater, ${ }^{7}$ olive oil mill effluents. ${ }^{8,9}$ Currently, industrial processes for PHA production are based primarily on the use of pure cultures of specific microorganisms, batch cultivation conditions and use of ad hoc formulated substrates (usually based on the imbalance between carbon and nitrogen). In order to reduce the PHA production costs and further increase its environmental sustainability, alternative processes have been proposed based on the use of waste organics as a substrate and of microbial mixed cultures for the biological PHA accumulation. The latter usually requires that microbial mixed cultures have been previously acclimated and/or selected to the substrate under periodic feeding, ${ }^{10-12}$ Typically, the proposed process includes three stages: the first stage, acidogenic fermentation of organic waste or wastewater (e.g. primary and/or secondary sludge) produces a concentrated mixture of organic acids needed to feed second and third stages. The second stage is performed under periodic feeding in order to acclimate, select and continuously produce a biomass with a high storage response that is then used in the third stage at a higher substrate load for further polymer accumulation.
The biomass selection in the second stage is usually obtained under alternating excess and lack of substrate conditions (feast and famine), done in aerobic sequencing batch reactor (SBR). ${ }^{13-15}$ Such conditions create a strong selective advantage in favor of those microorganisms that are both able (a) to most quickly consume the substrate (converting it into PHA) in the feast phase, and (b) to most quickly reuse the stored PHA back for cellular growth in the following famine phase.

With reference to $\mathrm{PHA}$-accumulating culture selection in SBR, previous studies were focusing on the mechanisms for increased selective pressure for PHA storage; the existing literature supports the hypothesis of a strong pressure for PHA storage being related to the feast/famine to which the cultures are exposed.

\footnotetext{
Correspondence to:Francesco Valentino, Department of Chemistry, "Sapienza", University of Rome, P.le Aldo Moro 5, 00185 Rome, Italy.

E-mail: francesco.valentino@uniroma1.it

a Department of Chemistry, Sapienza University of Rome, P.le Aldo Moro 5, 00185 Rome, Italy

b Department of Civil, Environmental and Materials Engineering (DICAM), University of Bologna, via Umberto Terracini 28, 40131 Bologna, Italy

c Interuniversity Consortium "Chemistry for the Environment" (INCA), Via delle Industrie 21/8, 30175 Venezia - Marghera (VE), Italy
} 
Johnson et al. ${ }^{16}$ maximized the selective pressure imposed on the culture selection SBR (using an influent acetic acid concentration of $54 \mathrm{mmol} \mathrm{L}^{-1}$ ) by gradually decreasing the sludge retention time (SRT) to 1 day and gradually increasing the operating temperature to $30^{\circ} \mathrm{C}$. The authors obtained a culture with a high storage capacity (89\% PHA content under nitrogen limitation during the accumulation step). The relative low SRT and high $\mathrm{T}$ are suggested by the authors to be, at least, partially responsible for the high kinetic parameters reported. In addition, the influent substrate concentration resulted in a very low feast phase ( $9 \%$ of the overall cycle length).

Dionisi et al. ${ }^{14}$ tested (through change in influent substrate concentration) the effect of the organic load rate (OLR) on the performance of an SBR fed with a mixture of synthetic organic acids (lactic, acetic and propionic acids), in the range of OLR 8.5-31.25 gCOD $L^{-1} d^{-1}$. The authors reported a high storage response for less high OLRs (feast phase up to $22.5 \%$ ), a growth response for higher OLRs (feast phase higher than 50\%), and a transient response at an intermediate value in which a very unstable system was selected (feast phase varying from 22.5 to $50 \%$ ).

In a later study, ${ }^{13}$ the authors operated the SBR (fed with a synthetic mixture of acetic and propionic acid) at very short HRT in the range 1-0.21 day. It was observed that, at decreasing HRT, the feast phase increased from $16.7 \%$ to $64 \%$, with a progressive loss of the selective pressure required to select microorganisms with high storage response (e.g. PHA specific storage rate decreased from 389 to $84 \mathrm{mgCOD} \mathrm{g}^{-1}$ CODnonPolym $\mathrm{h}^{-1}$, respectively).

In a study by Albuquerque et al., ${ }^{1}$ at lower OLRs (2.2-4.4 gCOD $\mathrm{L}^{-1} \mathrm{~d}^{-1}$, using fermented real wastewater), the range of feast phase length observed was $17-50 \%$, consistent with the findings of Dionisi et al. ${ }^{14}$ the lowest OLR resulted in feast length of $17 \%$ and was associated with a predominantly storage response, while a very unstable system (highly dynamic) with alternate predominantly storage and growth responses was obtained for a higher influent substrate concentration, the resulting feast phase length varying for the same average loading from $34.6 \%$ to $52 \%$ of the overall cycle length.

The SBR is usually inoculated by an activated sludge from wastewater treatment plants, whose ability to produce PHAs is progressively increased through several mechanisms (physiological adaptation, selection and enrichment). Thus, the quickness of activated sludge adaptation before reaching high PHA storage response is also a key factor for process design and operation as well as for process control, also taking into account possible disturbances from the steady state. Very few studies have focused on the acclimation phase of the biomass under new dynamic feeding conditions and new types of substrate, both different from wastewater treatment plants. Mengmeng et al. ${ }^{17}$ investigated the storage capacity of no-acclimated biomass taken from the municipal wastewater treatment system. Their experimental plan did not include any acclimation or enrichment stage, as usually done in order to obtain a good selection of biomass with high storage response. The biomass consisted of excess sludge sampled from the secondary sedimentation tank of a wastewater treatment plant; it was directly inoculated into an aerobic batch reactor where an excess sludge fermentative liquid (characterized by high VFAs content) was used as substrate. The sludge accumulated PHA immediately and the intracellular PHA content increased from $3.5 \%$ to $26.7 \%$ of the dry cell before the second substrate feeding. This indicated rapid biomass adaptation to the new conditions. Spitting the substrate in three pulses led to $56.5 \%$ of PHA content at the time of total substrate depletion ( $3 \mathrm{~h}$ and $40 \mathrm{~min}$ ). This saturation value, confirmed by no increase of PHA content observed after the fourth pulse, was obtained by using real wastes as carbon source and activated sludge as inoculum without sludge acclimation.

Based on the lack of knowledge related to the biomass behavior in the acclimation phase, under a new dynamic feeding condition in SBR (feast and famine), in the present study we investigated the process performance in the early days after SBR start up (from 2 to 10 days from the SBR inoculation) by taking the biomass from the SBR during start up and performing the third accumulation stage in batch experiments under pulse feeding conditions. A molecular analysis of the microbial composition of the mixed culture which developed in a long-term SBR run was also carried out by means of denaturing gradient gel electrophoresis (DGGE) and PCR amplification of the bacterial 16S rRNA genes. This was done in order to evaluate any changes occurring in the structure of the bacterial community throughout the run, from the start-up to the end.

\section{MATERIALS AND METHODS \\ Acclimation and selection stage (SBR run)}

The aerobic enrichment of the PHA-producing biomass was performed in a $1 \mathrm{~L}$ working volume SBR that was inoculated with the activated sludge from the 'Roma Nord' full-scale wastewater treatment plant. Before each inoculum the sludge was left to settle in order to remove the supernatant, and resuspended in a synthetic mineral medium (whose the composition is given below). Then, the sludge was aerated for at least one night before the inoculum; an average volatile suspended solid (VSS) concentration of $4 \mathrm{~g} \mathrm{~L}^{-1}$ was measured.

After each inocula, the SBR was fed with a mixture of acetic and propionic acid ( $85 \%$ and $15 \%$ on a COD basis, respectively), at an overall concentration of $8.5 \mathrm{gCOD} \mathrm{L}^{-1}$. The SBR was operated at 12 cycles per day (cycle length $2 \mathrm{~h}$ ); the substrate feeding and the mixed liquor discharge were made for the first $10 \mathrm{~min}$ and for last $1 \mathrm{~min}$ of each cycle. Overall, the SBR was operated at applied organic load rate (OLR) $8.5 \mathrm{gCOD} \mathrm{L}^{-1} \mathrm{~d}^{-1}$ and hydraulic residence time (HRT) of 1 day. No settling phase was performed, and all excess biomass was withdrawn along with the mixed liquor. In this way, the biomass retention time was equal to hydraulic retention time (HRT of 1 day).

Four SBR runs were carried out sequentially under the same operating conditions and for a relatively short period (20 days maximum). An additional run was started, under the same operating conditions as previously but characterized by a much longer period (more than 150 days) in order to reach complete sludge acclimation to the feast-famine regime, to check process stability and to compare the storage performance with that of the rapidly acclimated sludge. The inocula were sampled from the wastewater treatment plants and used fresh each time the reactor was started again. The reactor was stirred by a mechanical impeller and aerated by means of membrane compressors (in order to maintain aerobic condition). On work days, the reactor was cleaned daily during the famine phase, by passing manually a spatula along the walls in order to prevent the biofilm formation and to obtain homogeneous conditions inside the reactor. Reactor cleaning was not performed during the week-end, this involved some biomass growth on the SBR walls.

The organic feed was prepared in a mineral medium, all the elements being present in excess of biomass requirements for growth (thus preventing any external limitation to growth). 
The mineral medium concentration was $\left(\mathrm{mg} \mathrm{L}^{-1}\right)$ : $\left(\mathrm{NH}_{4}\right)_{2} \mathrm{SO}_{4}$ (3000), $\mathrm{FeCl}_{3} \cdot 6 \mathrm{H}_{2} \mathrm{O}$ (2), $\mathrm{Na}_{2} \mathrm{EDTA}$ (3), $\mathrm{K}_{2} \mathrm{HPO}_{4}$ (330), $\mathrm{KH}_{2} \mathrm{PO}_{4}$ (260), $\mathrm{MgSO}_{4} \cdot 7 \mathrm{H}_{2} \mathrm{O}(100), \mathrm{CaCl}_{2} \cdot 2 \mathrm{H}_{2} \mathrm{O}(50), \mathrm{ZnSO}_{4} \cdot 7 \mathrm{H}_{2} \mathrm{O}$ (0.1), $\mathrm{MnCl}_{2} \cdot 4 \mathrm{H}_{2} \mathrm{O}(0.03), \mathrm{H}_{3} \mathrm{BO}_{3}(0.3), \mathrm{CoCl}_{2} \cdot 6 \mathrm{H}_{2} \mathrm{O}$ (0.2), $\mathrm{CuCl}_{2} \cdot 2 \mathrm{H}_{2} \mathrm{O}(0.01), \mathrm{NiCl}_{2} \cdot \mathrm{H}_{2} \mathrm{O}(0.02), \mathrm{Na}_{2} \mathrm{MoO}_{4} \cdot 2 \mathrm{H}_{2} \mathrm{O}(0.03)$. Thiourea $\left(20 \mathrm{mg} \mathrm{L}^{-1}\right)$ was also added to inhibit nitrification. The $\mathrm{pH}$ was maintained at 7.5 by dosing $\mathrm{CO}_{2}$, which was suitably added via a control system. The temperature was maintained at $25^{\circ} \mathrm{C}$ using a thermostatic bath.

During each SBR cycle, the dissolved oxygen (DO) was continuously monitored and recorded using a DO probe (CellOx 325 WTW). Aeration and stirring were sufficient to maintain DO above $2 \mathrm{mg} \mathrm{L}^{-1}$ throughout the whole cycle. During feeding, the DO concentration decreased to a low and almost constant value; the following increase of DO concentration indicated that the substrate had been depleted. This point also indicated the end of the feast phase and the start of the famine phase and was used to calculate the feast-famine ratio for each cycle. The SBR performance was also monitored by measurement of biomass concentration, as volatile suspended solid (VSS, sample taken at the end of the feast phase), and of PHA (samples taken at the end of both the feast phase and the famine phase). At the end of the feed the maximum value of substrate concentration within each cycle was obtained, whereas the time of substrate depletion corresponded to the maximum value of polymer concentration. Sampling was usually done once per day.

\section{Batch accumulation tests}

For each short SBR run, two or three short-term aerobic batch tests were performed in the period ranging from 2-10 days from the start-up (i.e. presumably when biomass was not completely acclimated). For the longest SBR run, two similar batch tests were conducted after approximately 150 days, a more than adequate period to achieve stable operating conditions and a high biomass storage response. All the batch tests were performed with multiple and instantaneous spikes of substrate in order to obtain a feast phase longer than in the SBR and so to determine the maximum specific PHA storage rates, the PHA storage yield, the maximum polymer content achievable in the biomass and the polymer produced at the end of the batch accumulation test. The biomass was withdrawn from SBR at the end of the feast phase (when the content of PHA was high), put in a smaller reactor (working volume $500 \mathrm{~mL}$, same temperature and $\mathrm{pH}$ as the second stage), diluted four times with the same mineral solution used for the SBR, with the only difference that it did not contain any nitrogen source, in order to prevent cellular growth and to increase the achievable polymer content. Thus, the only nitrogen available at the beginning of the tests was the residual ammonia content in the mixed liquor withdrawn from the SBR. The diluted biomass was then spiked with multiple additions of the same substrates fed to the second stage in order to saturate the biomass storage capacity; the initial substrate concentration was $1000 \mathrm{mgCOD} \mathrm{L}^{-1}$. The mixed liquor in the reactor was sampled at regular intervals for analytical determination of the substrates, $\mathrm{PHA}$, and ammonia. Before (at least $20 \mathrm{~min}$ ) and during the test, the batch reactor was maintained under air bubbling (at oxygen concentrations in the range $7-8 \mathrm{mg} \mathrm{L}^{-1}$ ). In order to measure the oxygen uptake rate (OUR), aeration was interrupted at intervals and the dissolved oxygen decrease was measured as a function of time (however, oxygen concentration never decreased at concentration lower than $2 \mathrm{mg} \mathrm{L}^{-1}$ ).

\section{Analytical methods}

The substrates (acetate and propionate) were measured on filtered samples $(0.45 \mu \mathrm{m}$ porosity) by gas chromatography (stationary phase Carbowax $20 \mathrm{~mol} \mathrm{~L}^{-1} 4 \%$ on CarboPack B-DA on a PerkinElmer 8410 instrument). Ammonium ions were measured on filtered samples by Nessler spectrophotometric method (the absorbance of the coloured complex was measured at $410 \mathrm{~nm}$ wavelength; Varian DMS 90 UV-Visible Spectrophotometer).

For PHAs determination, the mixed liquor sample was treated immediately with a $\mathrm{NaClO}$ solution (7\% active $\mathrm{Cl}_{2}$ ) and stored at $-4{ }^{\circ} \mathrm{C}$ for the following analysis. PHAs were extracted, hydrolysed and esterified to 3-hydroxyacyl methyl esters, and determined by gas chromatography. ${ }^{18}$ The relative abundance of HB and $\mathrm{HV}$ monomers were quantified by using a commercial polymer $\mathrm{P}(\mathrm{HB} / \mathrm{HV}$ ) of known HV content, as standard (Poly(3-hydroxybutyric acid-co-3-hydroxyvaleric acid), PHV content 12\% wt, SigmaAldrich).

The non-polymer biomass (active biomass $X_{A}$ ) was calculated from the difference between VSS and PHA in the sample and converted into COD according to a conversion factor of 1.42 mgCOD mg $^{-1}$ biomass: $\mathrm{X}_{\mathrm{A}}=(\mathrm{VSS}-\mathrm{PHA}) 1$.42. The latter conversion factor was obtained by considering the generic heterotrophic biomass formula $\mathrm{C}_{5} \mathrm{H}_{7} \mathrm{O}_{2} \mathrm{~N} .{ }^{19}$

PHAs were also converted into COD according to oxidation stoichiometry: $1.67 \mathrm{mgCOD}^{-1} \mathrm{HB}$ monomer, and $1.92 \mathrm{mgCOD}$ $\mathrm{mg}^{-1}$ HV monomer. The PHAs content of the biomass was calculated by dividing the measured PHA concentration by biomass concentrations (both expressed as COD).

\section{Calculations}

In the SBR, the amount of stored $\mathrm{PHA}(\triangle \mathrm{PHA})$ was calculated as the difference between PHA concentration in the mixed liquor at the end of the feast phase (i.e. the substrate depletion time) and at the start of the respective cycle. The specific PHA production rate was calculated as the ratio of stored PHA and the length of feast phase $(t)$, per unit of non-polymer biomass $\left(X_{A}\right)$ : $r P H A=\triangle P H A /\left(t X_{A}\right)$. The storage yield during the feast phase was determined as the ratio between the amount of stored PHA (as COD) and the amount of removed substrate (as COD) fed in the cycle: $\mathrm{Y}_{\text {STO }}=\Delta \mathrm{PHA} / \Delta \mathrm{S}$. The observed yield was determined as the ratio between the non-polymer biomass $\left(\mathrm{X}_{\mathrm{A}}\right)$ concentration at substrate depletion, and the amount of substrate (as COD) fed in the cycle: $Y_{O B S}=X_{A} / \Delta S$. The polymer content in the biomass was calculated (in terms of COD) at substrate depletion time as the ratio of PHA concentration and volatile suspended solid concentration (i.e. the sum of non-polymer biomass and produced polymer): $\% \mathrm{PHA}=\Delta \mathrm{PHA} / \mathrm{VSS}=\Delta \mathrm{PHA} /\left(\mathrm{X}_{\mathrm{A}}+\Delta \mathrm{PHA}\right)$.

In batch tests the specific rates (storage and substrate consumption) were calculated by linear regression of data versus time, with reference to the first substrate addition only, in order to not include possible rate decrease due to saturation effects. For each batch test, the biomass concentration was calculated from the dilution ratio from the SBR to the batch test $(1: 4)$ and it was considered constant at its initial value, since the formation of new active biomass was practically negligible due to low growth yield and nitrogen limitation. The maximum polymer content in the biomass obtained during each test was also calculated based on PHA profiles; the storage yield was calculated only relative to the time when the maximum polymer content was achieved.

Based on results from short-term SBR runs and related batch tests, a hypothetical PHA productivity (gPHA $\mathrm{L}^{-1} \mathrm{~d}^{-1}$ ) was 
calculated by considering a novel scenario where the whole biomass could be used for PHA accumulation, by feeding the SBR at a higher substrate load and harvesting the whole biomass from the SBR at the end. Hence, the PHA productivity was calculated from the final PHA concentration $\left(\mathrm{g} \mathrm{L}^{-1}\right)$ achievable in the SBR (as estimated from batch test results) divided by the SBR volume $(1 \mathrm{~L})$ and the time passed. The latter was calculated by the sum of the time passed from SBR inoculum when the biomass was sampled and the batch test duration. The PHA productivity when using two separate reactors for II and III stages was calculated elsewhere. ${ }^{13}$

\section{DGGE analysis of the bacterial community during acclimation} The structure of the bacterial community was investigated throughout the biomass adaptation associated with the long SBR run lasting more than 150 days. Samples of biomass were collected from the reactor at the beginning (day 0 ) and after 1, 2, 7, 30, 50, 120 and 154 days of process operation and stored at $-20^{\circ} \mathrm{C}$ in $50 \%(\mathrm{v} / \mathrm{v})$ ethanol. Metagenomic DNA was extracted from approximately $250 \mathrm{mg}$ of pellet with the UltraClean Soil DNA kit (MoBio Laboratories, Carlsbad, CA, USA) according to the manufacturer's instructions. PCR amplification of the bacterial 16S rRNA genes of the community was performed with primers GC-357f and $907 r^{20}$ as described elsewhere. ${ }^{21}$ PCR products were resolved with a D-Code apparatus (Bio-Rad, Milan, Italy) on a $7 \%(\mathrm{w} / \mathrm{v})$ polyacrylamide gel (acrylamide- $\mathrm{N} \mathrm{N}^{\prime}$-methylenebisacrylamide, $37: 1$ ) in $1 \times \mathrm{TAE}$ with a denaturing gradient from $40 \%$ to $60 \%$ denaturant, where $100 \%$ denaturant is $7 \mathrm{~mol} \mathrm{~L}^{-1}$ urea and $40 \%(\mathrm{v} / \mathrm{v})$ formamide. The electrophoresis was run at $90 \mathrm{~V}$ for $15 \mathrm{~h}$ at $60^{\circ} \mathrm{C}$. The gel was stained in a solution of $1 \times$ SYBR Green I (Sigma-Aldrich, Milwaukee, WI) in $1 \times$ TAE for 30 min and its image captured in UV transillumination with a digital camera supported by a Gel Doc apparatus (BioRad, Milan, Italy). Subsequently, a matrix of similarities between the densitometric curves of the band patterns was calculated based on the Dice coefficient with Quantity One 4.5.2 software (Bio-Rad). Finally, the DGGE patterns were clustered based on the unweighted pair-group arithmetic average (UPGAMA) clustering algorithm.

\section{RESULT}

\section{SBR runs (biomass adaptation)}

Short-term SBR runs

Figure 1 (B) shows the length of the feast phase (substrate depletion time) of each cycle during the four short SBR runs. As reported above, the length of feast phase corresponds to the time of substrate depletion, as easily indicated from the oxygen profile during each cycle (Fig. 1(A)). In general a wave sequence of alternating short and long feast phases was observed for runs I-III: indeed, feast phase length was changing in the range 15-120 min (in the latter case, being equal to the overall cycle length) with no single monotonic trend from long to short feast phase. On the other hand, the intensity of the wave pattern decreased with time, showing a tendency to stabilize down to a shorter feast phase length. The only exception is run IV, which was stopped during the first peak and so before the expected new decrease of feast phase length. The length of each SBR run was not defined in advance; it was established based on the observed profiles of the feast phase length, in order to make it possible to carry out batch tests corresponding to high and low values of feast phase length.

Even though a general trend was observed, from Fig. 1(B) it is also clear that the observed trends were different and poorly reproducible. The observed difference among these short SBR runs can probably be attributed to the dynamic response of the different inocula under the imposition of strong variations of feed conditions with respect to previous ones in the full scale plants. The poor reproducibility of the observed trends can be explained by the inevitable differences among the inocula used in the four SBR runs. Indeed, the inocula are complex ecosystems sensitive to the fluctuations of operational factors (such as hydraulic regime, $\mathrm{pH}$, temperature, wastewater, composition, etc.) that occur in activated sludge wastewater treatment plants. Consequently, the tested inocula show different abilities to adapt themselves to the strong variation of feeding conditions passing from the full scale plants to the SBR. The short duration of the runs in SBR amplified the differences in adaptation shown by the different inocula.

Even though new feed conditions were the same for the four SBR, it can be supposed that sudden and strong variations amplified small differences among the different inocula. As an example, run III was characterized by a more stable behavior with respect to runs I and II.

As the length of feast phase changed irregularly, the selective pressure in favor of PHA storing microorganisms varied during each SBR run. Accordingly, Fig. 2(A) shows the time profile of specific storage rate during the feast phase for the different SBR runs. The values obtained varied over a wide range between 4 and $361 \mathrm{mgCOD} \mathrm{g}^{-1} \mathrm{CODnonPolym} \mathrm{h}^{-1}$, one or two peaks of high storage rate appearing in each run. Again, the time profiles of storage rate were different in each SBR run and related peaks were also shifted in time between the different runs. This confirms the poor reproducibility of process performance in the startup period, early after each inoculation with activated sludge. Figure 2(B) shows that the polymer content in the biomass at the end of feast phase was also widely variable during each run and from one run to the another one. As an example, in Run I the maximum polymer content appeared after 5 days from the start-up (29\% COD $\left.\operatorname{COD}^{-1}\right)$; in Run II and III, PHA peaks were very similar each other but at a quite lower value and shifted 2 days earlier than Run I; in Run IV the maximum polymer content were obtained after 9 days from the inoculum, immediately before the run was stopped. These variations were probably because under the feast and famine regime, the biomass needs to adapt itself to both store intracellular PHA during the feast phase and to reuse PHA during the famine phase. It can be argued that adaptation to store PHA is quicker than to reuse PHA; hence PHA are initially accumulated inside cells and this in turn can decrease the substrate uptake rate in the following cycles. ${ }^{13}$ Considering the high performance variability observed for all four runs during SBR start up, average values and relative standard deviation of monitored parameters were not calculated.

\section{Long-term SBR run}

In order to better interpret such a high variability of short-term SBR runs, a fifth SBR run was started and performed for a much longer period. Figure 3 shows the trend of the feast phase length as a function of days passed from inoculum, during the longterm SBR run. Even in this case, the unstable process performance immediately after the inoculum is evident. However, the frequency and intensity of oscillating length of the feast phase strongly decreased with time, likely because of the ongoing acclimation of the biomass under new dynamic feeding. An almost stable SBR performance was achieved after $\sim 15$ days (180 cycles) from the initial inoculum; the residual small fluctuations observed during the pseudo-steady state were almost totally correlated with the 

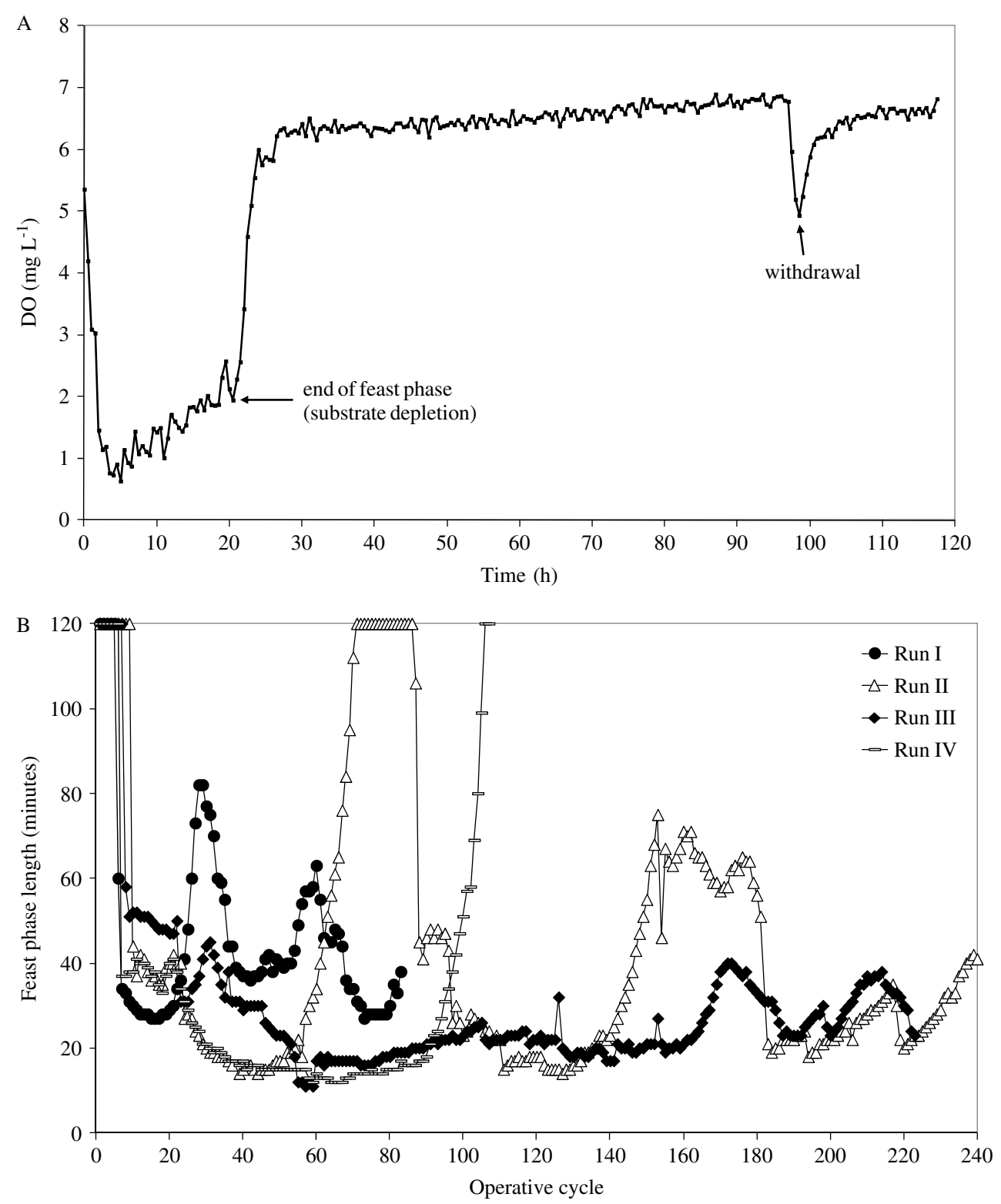

Figure 1. (A) Typical oxygen profile from which the length of feast phase was calculated. (B) Time profiles of length of the feast phase during the four short SBR runs.

lack of SBR cleaning at each week-end (with formation of some biofilm on the SBR glass walls, see Material and methods). Figure 4 (A),(B) shows the specific storage rate and the polymer content in the biomass at the end of feast phase during the long-term SBR run: the storage rate trend confirmed the high performance instability during the initial acclimation phase, whereas much smaller fluctuations were observed for the rest of the run (although if higher values were obtained just at the end of the SBR run).

As for PHA content in the biomass a quite high polymer content (58\% COD $\mathrm{COD}^{-1}$ ) was observed after only 3 days from the inoculum, confirming the hypothesis of a quicker adaptation to store PHA in the feast phase than to reuse PHA in the famine phase. Then, the PHA content stabilized at around $10 \%$ for the rest of the SBR run.

Table 1 summarizes average values of the measured parameters in this run ('steady state' period from day 15 to day 154): the feast phase percentage was less than $20 \%$ of the overall cycle length; this guaranteed an adequate selective pressure on the PHA-storing microorganisms. This was confirmed by the good storage capacity during the feast phase: the specific storage rate was high (190 mgCOD gCODnonPolym $\left.{ }^{-1} \mathrm{~h}^{-1}\right)$ as well as the storage yield $(0.32$ $\operatorname{COD}\left(\mathrm{COD}^{-1}\right)$. It is worthwhile mentioning that this good storage performance was obtained during the feast phase with no nitrogen limitation.

As for microbiological analysis, remarkable changes occurred in the structure of the bacterial community throughout the run (Fig.5). In particular, DGGE patterns at days 0,1 and 2 were highly similar to each other and sub-clustered together within a cluster including day 7. In addition, strong similarity was observed between patterns at days 30 and 50 and patterns at days 120 and 154, which sub-clustered separately and clearly distinguished themselves from patterns at days $0-7$. This indicates that the inoculum progressively evolved throughout the SBR run. The quickest variation of microbial composition in the period between 

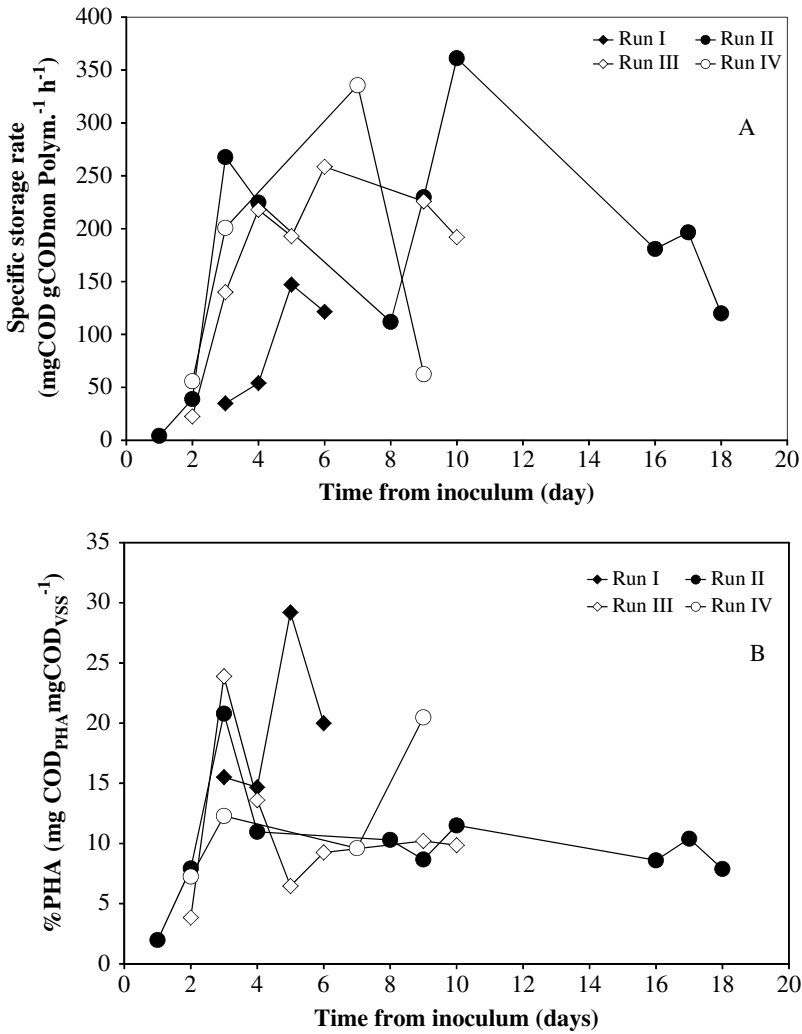

Figure 2. Specific storage rate $(A)$ and polymer content in the biomass (B) at the end of the feast phase in all short SBR runs during start-up.

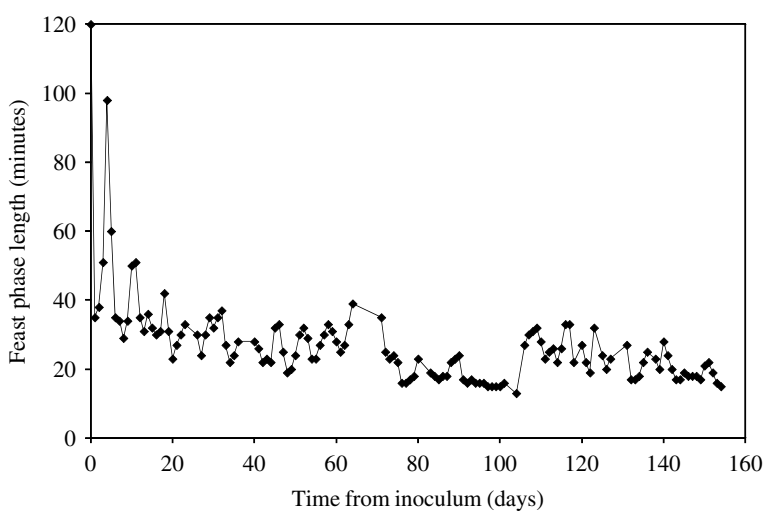

Figure 3. Length of feast phase during the long SBR run.

the inoculum and day 7 well reflects the higher variability of SBR performance during start up. Changes of the structure of the microbial community lasted during the 'steady state' period, even though at a lower rate, but they did not cause substantial changes of PHA storage rate; e.g. very similar specific storage rates were observed at days 30, 50 and 120 ( 166-189 mgCOD gCODnonPolym $\left.{ }^{-1} \mathrm{~h}^{-1}\right)$. On the other hand, at day 120 the PHA storage rate was more similar to days 30-50 despite the microbial community structure being more similar to that at day 154, when the PHA storage rate showed a new peak. At days 2 and 154 the highest storage rate values were obtained (350 and 284 mgCOD gCODnonPolym ${ }^{-1} \mathrm{~h}^{-1}$ respectively), in spite of the very low similarity of the respective microbial communities.
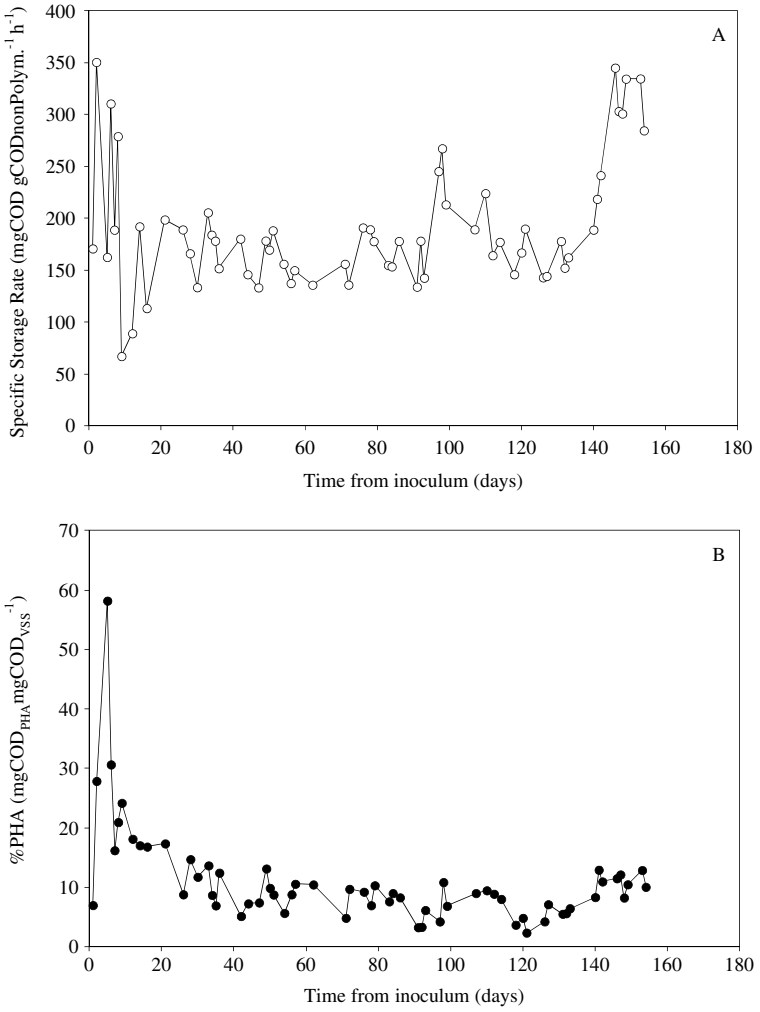

Figure 4. Specific storage rate $(A)$ and polymer content in the biomass (B) at the end of the feast phase during the long SBR run.

Table 1. Average values during pseudo-steady-state of measured parameters in long term SBR run

Parameter

(SBR, Run, OLR 8.5gCODL ${ }^{-1} \mathrm{~d}^{-1}, \mathrm{HRT} 1$ day, overall cycle length $2 \mathrm{~h}$ )

nonPolymer Biomass concentration at substrate depletion $\left(\mathrm{mgCODL}^{-1}\right)$

PHA concentration at the end of cycle $\left(\mathrm{mgCODL}^{-1}\right) \quad 124 \pm 7$

PHA concentration at substrate depletion $\quad 374 \pm 13$ $\left(\mathrm{mgCODL}^{-1}\right)$

feast phase length (min.)

$23.6 \pm 0.6$

feast/famine ratio (\%)

$19.7 \pm 0.5$

specific substrate removal rate (mgCOD

$612 \pm 26$

gCODnonPolym. ${ }^{-1} \mathrm{~h}^{-1}$ )

specific storage rate (mg COD gCODnonPolym. ${ }^{-1} \mathrm{~h}^{-1}$ )

$190 \pm 7$

Observed Yield $\left(\mathrm{Y}_{\mathrm{OBS}}^{\mathrm{SBR}} ; \mathrm{COD}^{\mathrm{C}} \mathrm{COD}^{-1}\right)$

$0.40 \pm 0.01$

Storage Yield ( $\mathrm{Y}_{\text {STO }}^{\text {feast }}$; $C O D$ COD $^{-1}$ )

$0.32 \pm 0.01$

These results suggest that the capability to store PHAs was not strictly correlated with the structure of the bacterial community, but also that feast and famine conditions were anyway able to steadily select for PHA-storing microbial species with high PHA storage capabilities.

\section{Batch tests from SBR run (PHA accumulation)}

Effect of acclimation time to 'feast and famine' regime during start-up During each of short SBR runs (I-IV), two or more batch tests were conducted with biomass sampled from the SBR at a prechosen cycle. The SBR cycles in which the biomass was sampled were characterized by a wide range of feast phase lengths (from 


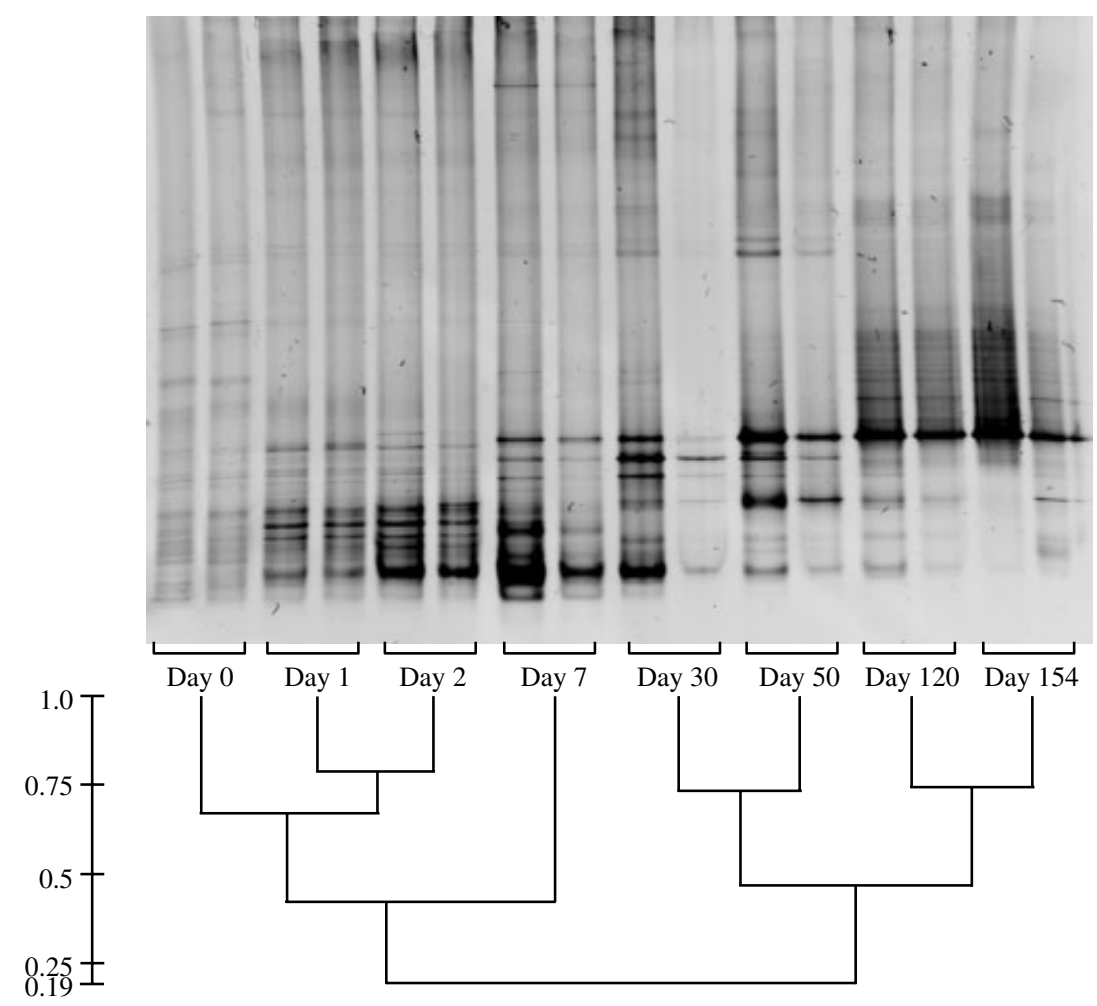

Figure 5. Structure of the bacterial community throughout the long-term SBR run. DGGE patterns were clustered with the unweighted pair-group arithmetic average (UPGAMA) clustering algorithm.

$12.5 \%$ to $88.3 \%$ of overall cycle length); this means that different selective pressure were existing at the time that biomass was sampled from each SBR run, regardless of the time passed from the initial inoculum.

As an example of batch test profiles, Fig. 6(A) shows the oxygen uptake rate (OUR), ammonia concentration and PHA content in the biomass in a batch test (Batch test IX) carried out sampling the biomass after 7 days from SBR start-up (IV SBR run) and during a cycle in which the substrate depletion time was 15 min (feast phase at $12.5 \%$ of the overall cycle length, corresponding to a strong selective pressure). The OUR shows a strong increase compared with the initial endogenous metabolism; after the first substrate addition, it continued to increase at a slower rate until the ammonia was depleted in the medium (more or less $2 \mathrm{~h}$ ). Following the next additions, it tends to decrease as a consequence of lack of nitrogen in the medium which prevented new biomass emergence and also due to the gradual saturation of storage capacity. Accordingly, the substrate uptake rate tends to decrease during the test as shown by the decrease of the slope after the following additions (Fig. 6(B)); the production of PHA almost entirely occurred in the first $4 \mathrm{~h}$ of the test (Fig. 6(B)), then it stopped likely because of exhausted biomass storage capacity as confirmed by the almost total invariance of the OUR. The specific storage rate was 330 mgCOD gCODnonPolym ${ }^{-1} \mathrm{~h}^{-1}$ (in the first $2 \mathrm{~h}$ of the test), i.e. the biomass continued to store the polymer at the same specific rate of the feast phase of the SBR cycle (day 7 of Run IV, Fig. 4(A)). The amount of produced polymer in this test was $1080 \mathrm{mgCOD}$ $\mathrm{L}^{-1}$, the maximum polymer content in the biomass was $55.8 \%$ COD $\operatorname{COD}^{-1}$ and the corresponding storage yield was 0.45 COD $\mathrm{COD}^{-1}$. These results confirmed the good storage properties of the biomass sampled from a SBR cycle that was characterized by a short feast phase. This means that the biomass quickly acclimated itself to the feast and famine condition imposed in the SBR.

In a batch test carried out after only 2 days from the previous one (test $X$, day 9, Run IV), the storage performance was significantly less. The specific storage rate was only $5.0 \mathrm{mgCOD}$ gCODnonPolym ${ }^{-1} \mathrm{~h}^{-1}$ and the amount of polymer produced at

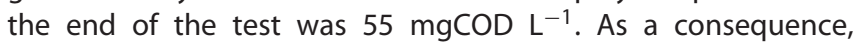
the maximum polymer content was $15.8 \% \mathrm{COD} \mathrm{COD}^{-1}$ and the corresponding storage yield $0.02 \mathrm{COD} C O D^{-1}$. Hence, after 9 days of acclimation the biomass response in the start-up period of SBR run was still unstable.

Table 2 summarizes the results obtained in all batch tests carried out during the four SBR runs in the period of start-up. These results confirm a high variability in performance that was completely unrelated to the time passed from the SBR inocula. On the contrary, a correlation between the length of feast phase in the SBR cycle in which the biomass was sampled and storage properties exhibited in the corresponding batch tests was clearly found. Regardless of the day during SBR start-up at which biomass was sampled, Fig. 7 shows that best storage performances were obtained when the feast phase of the biomass sampling cycle was low (at least $\leq 20 \%$ of the overall cycle length). The best storage performances were reached in four batch tests characterized by short feast phases (Test III, VII, VIII, IX) that showed the highest values of specific storage rate (in the range 182 to $330 \mathrm{mgCOD}$ gCODnonPolym $\left.{ }^{-1} \mathrm{~h}^{-1}\right)$, storage yield $\left(0.44-0.49 \operatorname{CODCOD}^{-1}\right)$ and

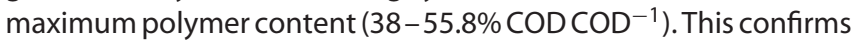
that a strong selective pressure on the biomass in the SBR (optimal regime of feast and famine) was necessary to obtain high storage response.

In the tests characterized by a longer feast phase of the biomass sampling cycle, the exhibited storage properties were 

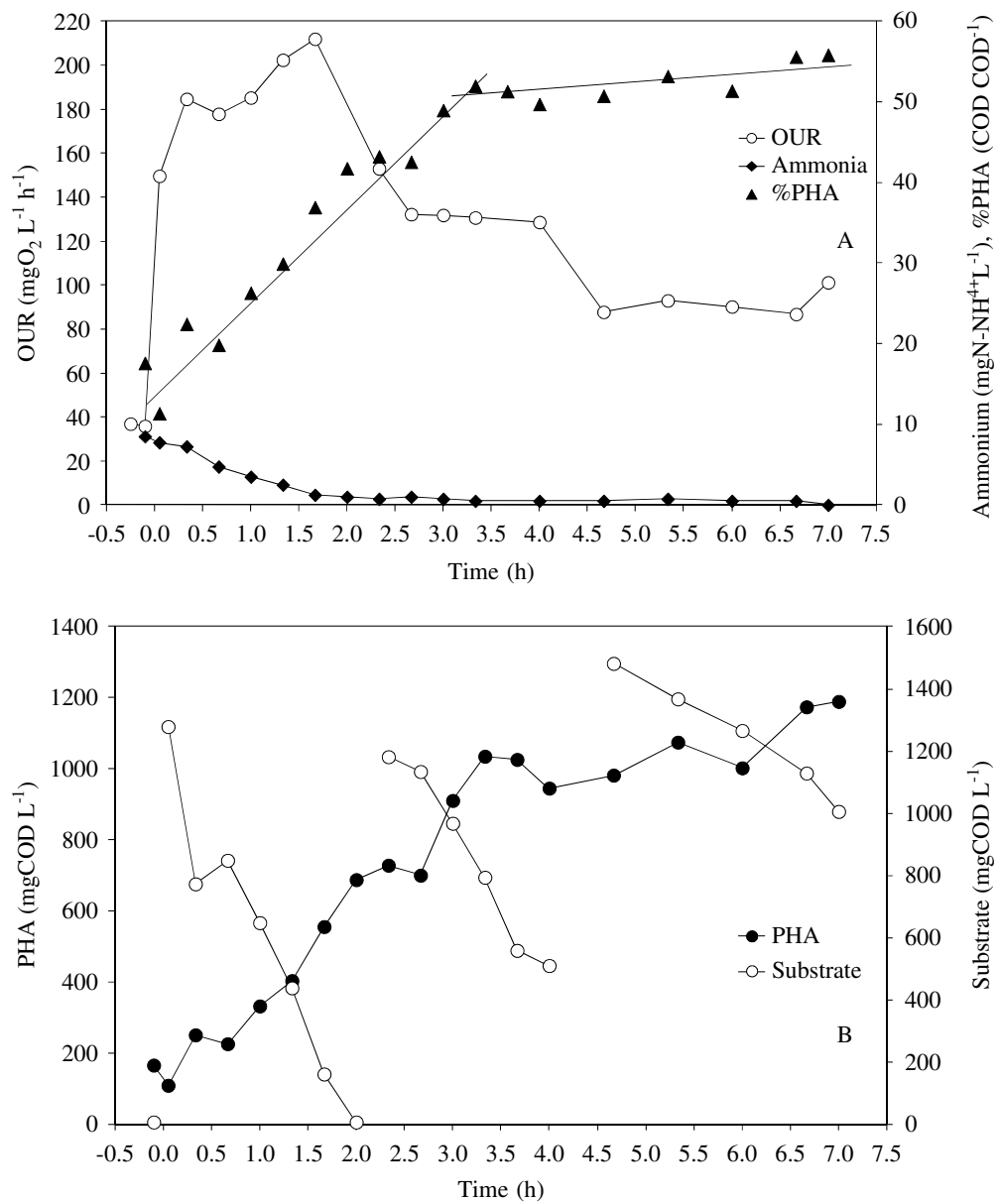

Figure 6. OUR, ammonia concentration and \%PHA (A); substrate and PHA concentration (B) in a batch test carried out with quickly acclimated biomass (run IV, batch test IX, biomass taken from a SBR cycle with very short feast phase).

Table 2. Summary of batch tests carried out during the different short SBR runs in unstable operating conditions

\begin{tabular}{|c|c|c|c|c|c|c|c|c|c|c|}
\hline \multirow[b]{3}{*}{ Parameter } & \multicolumn{10}{|c|}{ Partially acclimated biomass } \\
\hline & \multicolumn{2}{|c|}{ Run I } & \multicolumn{3}{|c|}{ Run II } & \multicolumn{2}{|c|}{ Run III } & \multicolumn{3}{|c|}{ Run IV } \\
\hline & Test I & Test II & Test III & Test IV & Test $\mathrm{V}$ & Test VI & Test VII & Test VIII & Test IX & Test $\mathrm{X}$ \\
\hline $\begin{array}{l}\text { Day of the SBR run (from the } \\
\text { inoculum) }\end{array}$ & 3 & 6 & 4 & 7 & 8 & 2 & 5 & 3 & 7 & 9 \\
\hline Biomass sampling cycle & 37 & 72 & 49 & 87 & 98 & 23 & 62 & 33 & 82 & 105 \\
\hline $\begin{array}{l}\text { feast phase length (biomass } \\
\text { sampling cycle, min.) }\end{array}$ & 44 & 30 & 17 & 106 & 30 & 31 & 16 & 20 & 15 & 99 \\
\hline $\begin{array}{l}\text { feast/famine ratio (biomass } \\
\text { sampling cycle, \%) }\end{array}$ & 36.7 & 25 & 14.2 & 88.3 & 25 & 25.8 & 13.3 & 16.7 & 12.5 & 82.5 \\
\hline $\begin{array}{l}\text { Specific storage rate }(\mathrm{rPHA} \\
\left.\mathrm{mgCODgCODnonPolym}^{-1} \mathrm{~h}^{-1}\right)\end{array}$ & 68 & 175 & 256 & 75 & 94 & 32 & 206 & 182 & 330 & 5 \\
\hline $\begin{array}{l}\text { Maximum polymer content }(\%, \\
\text { COD }_{\text {PHA }}\left(\operatorname{COD}_{\mathrm{VSS}}^{-1}\right)\end{array}$ & 22.1 & 41.4 & 48.7 & 25.6 & 32.3 & 14.8 & 38 & 48.4 & 55.8 & 15.8 \\
\hline Storage Yield $\left(\mathrm{Y}_{\mathrm{STO}}, \mathrm{COD}_{\mathrm{PHA}} \mathrm{COD}_{\Delta \mathrm{S}}^{-1}\right)$ & 0.09 & 0.23 & 0.44 & 0.26 & 0.18 & 0.1 & 0.49 & 0.48 & 0.45 & 0.02 \\
\hline Produced PHA $\left(\mathrm{mgCODL}^{-1}\right)$ & 209 & 594 & 895 & 139 & 301 & 129 & 662 & 756 & 1080 & 55 \\
\hline $\begin{array}{l}\text { Final PHA concentration } \\
\quad\left(\mathrm{mgCODL}^{-1}\right)\end{array}$ & 379 & 753 & 1061 & 267 & 455 & 189 & 734 & 866 & 1190 & 177 \\
\hline $\begin{array}{l}\text { Initial nonPolymer biomass } \\
\text { concentration }\left(\mathrm{Xa}, \mathrm{mgCODL}^{-1}\right)\end{array}$ & 841 & 887 & 863 & 625 & 890 & 1083 & 1088 & 785 & 854 & 637 \\
\hline
\end{tabular}



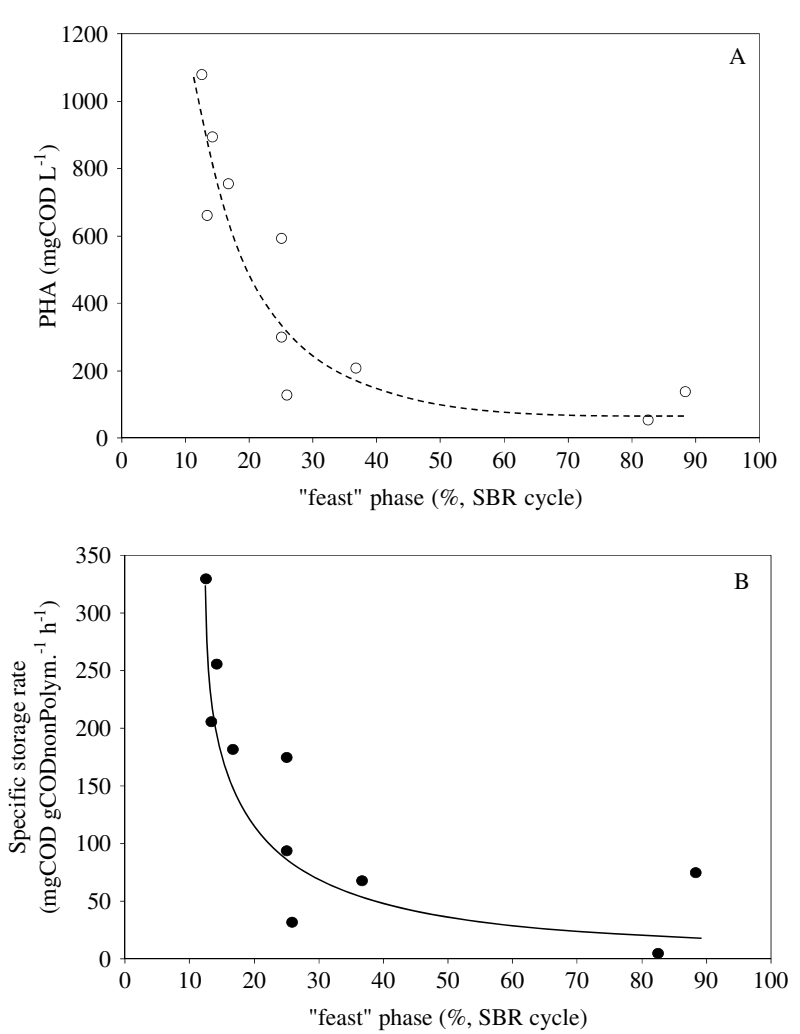

Figure 7. Polymer produced (A) and specific storage rate (B) in batch tests as a function of feast phase of the SBR cycle from which the biomass was sampled.

substantially lower (i.e. in tests I, II, V and VI, when the feast phase was in the range $25-36.7 \%$, too high to obtain a prevailing storage response). As a further example, Test IV and X, were conducted after 7 and 9 days from SBR start-up, respectively; hence the acclimation phase was longer than for batch tests III and VIII, which were carried out after 4 and 3 days from start-up, respectively. Despite the longer acclimation period, tests IV and $X$ showed a complete loss of biomass storage properties due to very long feast phase ( $88.3 \%$ and $82.5 \%$ of the overall cycle length, respectively). This means that at this high feast phase length, the selective pressure imposed on the biomass in the SBR was almost completely lost.
Overall, Table 2 and Fig. 7 confirm that the storage rate and yield exhibited in the batch tests were inversely correlated with the length of feast phase in the SBR cycle in which the biomass had been sampled whereas they were completely unrelated to the time passed from the SBR inocula.

\section{Effect of long acclimation time (pseudo-steady-state)}

The biomass response in the third stage was also studied through batch tests carried out with sludge sampling from the SBR under stable operating conditions (pseudo-steady-state).

The time profiles of the main parameters of accumulation tests carried out with fully acclimated biomass sampled from the longterm SBR run (not reported) were very similar to the profiles of Fig. $6(A),(B)$ that had been obtained with quickly acclimated biomass taken in a SBR cycle with very short feast phase.

Table 3 shows the average results from these batch tests and compares them with average results from tests performed with quickly acclimated biomass sampled during SBR start-up (only tests with short feast phase conditions were selected, i.e. tests III, VII, VIII and IX). Even though the biomass samples were characterized by quite different acclimation periods to the feast and famine conditions, their storage performance was very similar for specific storage rate, storage yield and maximum polymer content reached in the biomass. The key factor, common to these performances, was the short feast phase in the SBR cycle in which the biomass was sampled.

\section{CONCLUSION}

This study investigated how rapidly an activated sludge could acclimate to the newly imposed feast and famine regime (in a SBR), and how it affects the storage performance of acclimated biomass.

A short feast phase length during the SBR cycle (less than $20 \%$ of the cycle length) was the key parameter to obtain good selective pressure on PHA-storing microorganisms and consequently PHA storage at high rate and yield This condition was reached quickly, in no more than 40 cycles and no less than 22 cycles (3.33 and 2 HRTs, respectively). However, once reached for the first time, the short feast phase was not maintained and highly unstable SBR performance was observed for at least 8-10 days. In an additional long-term SBR run, a stable performance was obtained only after 15 days (15 HRT) and steadily maintained for a longer period.

Table 3. Range and mean values of the parameters calculated from batch tests carried out with quickly acclimated biomass (SBR start-up) and fully acclimated biomass (SBR pseudo-steady-state)

\begin{tabular}{|c|c|c|c|c|}
\hline \multirow[b]{2}{*}{ Parameters } & \multicolumn{2}{|c|}{$\begin{array}{l}\text { Quickly acclimated } \\
\text { biomass (SBR start-up) }\end{array}$} & \multicolumn{2}{|c|}{$\begin{array}{l}\text { Fully acclimated biomass } \\
\text { (SBR pseudo-steady state) }\end{array}$} \\
\hline & range & mean value & range & mean value \\
\hline Day of the SBR Run (from the inoculum) & $3-7$ & $4.8 \pm 0.9$ & $146-153$ & $150 \pm 4$ \\
\hline Biomass sampling cycle & $33-82$ & $57 \pm 10$ & $1746-1830$ & $1788 \pm 42$ \\
\hline feast phase length (biomass sampling cycle, min.) & $15-20$ & $17 \pm 1$ & $18-16$ & $17 \pm 1$ \\
\hline feast/famine ratio (biomass sampling cycle, \%) & $12.5-16.7$ & $14.2 \pm 0.9$ & $15-13.3$ & $14.2 \pm 0.8$ \\
\hline specific storage rate (mgCOD gCODnonPolym ${ }^{-1} \mathrm{~h}^{-1}$ ) & $182-330$ & $244 \pm 33$ & $183-209$ & $196 \pm 13$ \\
\hline Maximum polymer content $\left(\%, \mathrm{COD}_{\mathrm{PHA}} \mathrm{COD}_{\mathrm{VSS}}^{-1}\right)$ & $38-55.8$ & $48 \pm 4$ & $49.6-50.9$ & $50.3 \pm 0.7$ \\
\hline Storage Yield $\left(\mathrm{Y}_{\mathrm{STO}}, \mathrm{COD}_{\mathrm{PHA}} \mathrm{COD}_{\Delta \mathrm{S}}^{-1}\right)$ & $0.44-0.49$ & $0.47 \pm 0.01$ & $0.52-0.53$ & $0.53 \pm 0.01$ \\
\hline Produced PHA $\left(\mathrm{mgCODL}^{-1}\right)$ & $662-1080$ & $848 \pm 91$ & $852-897$ & $875 \pm 23$ \\
\hline Initial nonPolymer biomass concentration $\left(\mathrm{Xa}, \mathrm{mgCODL}^{-1}\right)$ & $785-1088$ & $897 \pm 66$ & $588-603$ & $595 \pm 7$ \\
\hline
\end{tabular}


It is worthwhile mentioning that the best storage properties exhibited by quickly acclimated biomass after 22-40 cycles were very similar to those obtained with fully acclimated biomass, characterized by a longer acclimation period. This evidence suggests a new strategy for operating the overall PHA production in a single reactor instead of using two separate reactors for biomass enrichment and the PHA accumulation, respectively. In this novel approach, the first achievement of a short feast phase in the SBR can be easily verified by on line monitoring of the dissolved oxygen profile. As soon as the short feast phase has been reached, the PHA accumulation stage is directly performed in the SBR, by feeding the biomass at a higher substrate load; finally, the whole biomass is harvested from the SBR for downstream processing (PHA extraction and purification). Based on present experimental data, the best PHA productivity was around $0.85 \mathrm{gPHA} \mathrm{L}^{-1} \mathrm{~d}^{-1}$ and final PHA content of $0.48 \mathrm{COD} \mathrm{COD}^{-1}$ basis (batch test VIII). Even though this value is lower than the best PHA productivity achievable using the usual two-reactor approach $(1.6 \pm 0.1 \mathrm{gPHA}$ $\mathrm{L}^{-1} \mathrm{~d}^{-1}$, as reported elsewhere ${ }^{13}$ ), the proposed approach is attractive because of its simplicity (a single reactor instead of two), also considering that this single reactor performance can be certainly optimized by investigating SBR start-up over a wider range of typical operating parameters (e.g. OLR, cycle length).

It is also noteworthy, that during the long-term SBR experiment similar performance was obtained even with low similarity of the structure of the microbial consortium. Cluster analysis of DGGE profiles showed that a slow drift of the microbial structure lasted for the whole long-term SBR run so that the good storage performance and the structure of microbial community were not fully correlated. In other words, these results indicate that feast and famine conditions were anyway able to steadily select for PHA-storing microbial species with high PHA storage capabilities, in spite of small changes in microbial structure.

This evidence can be relevant because changes of microbial population could in principle affect the molecular weight (MW) and other properties of PHA produced. Hence, the short-term acclimation used in this study does not necessarily mean a larger variability of PHA properties. More research is clearly needed on this topic, also depending on PHA end-uses, towards the perspective of full exploitation of PHA production from microbial mixed cultures.

\section{ACKNOWLEDGEMENTS}

This work was supported by the EU ROUTES project (Contract No 265156, FP7 2007-2013, THEME [ENV.2010.3.1.1-2] Innovative system solutions for municipal sludge treatment and management).

\section{REFERENCES}

1 Albuquerque MGE, Torres CAV and Reis MAM, Polyhydroxyalkanoates (PHA) production by a mixed microbial culture using sugar molasses: effect of the influent substrate concentration on culture selection. Water Res 44:3419-3433 (2010).

2 Albuquerque MGE, Eiroa M, Torres C, Nunes BR and Reis MAM, Strategies for the development of a side stream process for polyhydroxyalkanoate (PHA) production from sugar cane molasses. J Biotechnol 130:411-421 (2007).

3 Din MF Md, Ujang Z, van Loosdrecht MCM, Ahmad A and Sairan MF, Optimization of nitrogen and phosphorus limitation for better biodegradable plastic production and organic removal using single fed-batch mixed cultures and renewable resources. Water Sci Technol 53:15-20 (2006).

4 Salmiati Ujang Z, Salim MR, Din MF Md and Ahmad MA, Intracellular biopolymer productions using mixed microbial cultures from fermented POME. Water Sci Technol 56:179-185 (2007).

5 Bengtsson S, Werker A, Christensson M and Welander T, Production of polyhydroxyalkanoates by activated sludge treating a paper mill wastewater. Bioresource Technol 99:509-516 (2008).

6 Liu HY, Hall PV, Darby JL, Coats ER, Green PG, Thompson DE, et al, Production of polyhydroxyalkanoates during treatment of tomato cannery wastewater. Water Environ Res 80:367-372 (2008).

7 Mato T, Ben M, Kennes Cand Veiga MC, PHA production using brewery wastewater, in Proceedings of 4th IWA Specialized Conference on Sequencing Batch Reactor Technology (SBR4), Rome, pp.59-66 (2008).

8 Beccari M, Bertin L, Dionisi D, Fava F, Lampis S, Majone M, et al, Exploiting olive oil mill effluents as a renewable resource for production of biodegradable polymers through a combined anaerobic-aerobic process. J Chem Technol Biotechnol 84:901-908 (2009).

9 Dionisi D, Carucci G, Petrangeli Papini M, Riccardi C and Majone M, Olive oil mill effluents as a feedstock for production of biodegradable polymer. Water Res 39:2076-2084 (2005).

10 Dionisi D, Majone M, Papa V and Beccari M, Biodegradable polymers from organic acids by using activated sludge enriched by aerobic period feeding. Biotechnol Bioeng 85:569-579 (2004).

11 Morgan-Sagastume F, Karlsson A, Johansson P, Pratt S, Boon N, Lant P, et al, Production of polyhydroxyalkanoates in open mixed cultures from a waste sludge stream containing high levels of soluble organics, nitrogen and phosphorous. Water Res 44:5196-5211 (2010).

12 Ntaikou I, Kourmentza C, Koutrouli EC, Stamatelatou K, Zampraka A, Kornaros M, etal, Exploitation of olive oil mill wastewater for combined biohydrogen and biopolymers production. Bioresource Technol 100:3724-3730 (2009).

13 Beccari M, Majone M, Vallini G, Lampis S, Valentino F and Villano M, Effect of hydraulic and organic loads in a sequencing batch reactor on microbial ecology of activate sludge and storage of polyhydroxyalkanoates. Chem Eng Trans 20:187-192 (2010).

14 Dionisi D, Majone M, Vallini G, Di Gregorio Sand Beccari M, Effect of the applied organic load rate on biodegradable polymer production by mixed microbial cultures in a sequencing batch reactor. Biotechnol Bioeng 93:76-88 (2006).

15 Dionisi D, Majone M, Vallini G, Di Gregorio S and Beccari M, Effect of the length of the cycle on biodegradable polymer production and microbial community selection in a sequencing batch reactor. Biotechnol Progress 23:1064-1073 (2007).

16 Johnson K, Kleerebezem R, Muyzer G and van Loosdrecht MCM, Enrichment of a mixed bacterial culture with a high polyhydroxyalkanoate storage capacity. Biomacromolecules 10:670-676 (2009).

17 Mengmeng C, Hong C, Qingliang Z, Shirley SN and Jie R, Optimal production of polyhydroxyalkanoates (PHA) in activated sludge fed by volatile fatty acids (VFAs) generated from alkaline excess sludge fermentation. Bioresource Technol 100:1399-1405 (2009).

18 Braunegg G, Sonnleitner B and Lafferty RM, A rapid gas chromatographic method for the determination of poly- $\beta$ hydroxybutyric acid in microbial biomass. Eur J Appl Microbiol Biotechnol 6:29-37 (1978).

19 Gujer W and Henze M, Activated sludge modeling and simulation. Water Sci Technol 23:1011-1023 (1991).

20 Sass AM, Sass H, Coolen MJ, Cypionka H and Overmann J, Microbial communities in the chemocline of a hypersaline deepsea basin (Urania basin, Mediterranean Sea). Appl Environ Microbiol 67:5392-5402 (2001).

21 Zanaroli G, Balloi A, Negroni A, Daffonchio D, Young LY and Fava F, Characterization of the microbial community from the marine sediment of the Venice lagoon capable of reductive dechlorination of coplanar polychlorinated biphenyls (PCBs). J Hazard Mater 178:417-426 (2010). 